\title{
3 Research Square

\section{Patient-Reported Noninfectious Comorbidities in Chinese HIV-Infected Patients: The Gaps Among Self-Awareness, Diagnosis, and Prevalence}

Jun Chen

Shanghai Public Health Clinical Center

Hongzhou Lu ( $\square$ luhongzhou@fudan.edu.cn )

Shanghai Public Health Clinical Center

Shuiqing Liu

The Fifth People's Hospital of Guiyang

Jun Liu

The Third People's Hospital of Kunming

Haibo Ding

First Hospital of China Medical University

\section{Rugang Wang}

Affiliated Dalian Sixth People's Hospital, Dalian Medical University

Zhihao Meng

Longtan Hospital of Guangxi

Peng Zhang

The People's Hospital of Liuzhou City

\section{Yinghua Wei}

First Affiliated Hospital of GuangXi Medical University

Lijing Wang

Fifth Hospital of Shijiazhuang

Jie Yang

Yunnan Provincial Infectious Disease Hospital

Jian Zhang

Changchun Infectious Disease Hospital

Qingxia Zhao

Zhengzhou Sixth People's Hospital

\section{Xiaohong Chen}

Fourth Affiliated Hospital of Harbin Medical University

\section{Ruzhi Ou}

The Fourth People's Hospital of Nanning

\section{Yuanhong He}


Public Health Clinical Center of Chengdu

\section{Yahong Chen}

Mengchao Hepatobiliary Hospital

\section{Juan Chen}

The Second People's Hospital of Yancheng City

\section{Min Wang}

The First Hospital of Changsha

\section{Biao Zhu}

First Affiliated Hospital Zhejiang University

\section{Yaokai Chen}

Chongqing Public Health Medical Center

\section{Juan Jin}

The eighth hospital of Xi'an

\section{An Liu}

Beijing YouAn Hospital

\section{Yang Zhou}

The Third People's Hospital of Shenzhen

\section{Guijv Gao}

Beijing Ditan Hospital

\section{Ping Ma}

Tianjin Second People's Hospital

\section{Hongxia Wei}

Nanjing Second Hospital

\section{Feifei Su}

Wenzhou Central Hospital

\section{Lianguo Ruan}

Wuhan Medical Treatment Center

\section{Tianjun Jiang}

The Fifth Medical Center of PLAGH

\section{Nanhong Zheng}

Ningbo No. 2 Hospital

\section{Jianhua Yu}

Hangzhou Xixi hospital

\section{Guangyong Xu}

Qingdao Infectious Diseases Hospital

\section{Feng Qian}

The Fifth People's Hospital of Suzhou

\section{Cenmian Huang}

The Infectious Diseases Hospital of Gejiu city Yunnan Province 


\section{Lingyao Du}

West China Hospital of Sichuan University

\section{Na Wang}

Second Affiliated Hospital of Chongqing Medical University

\section{Huiling Zhou}

The First Peoples' Hospital of Foshan

\section{Jie Peng}

Nanfang Hospital

\section{Research Article}

Keywords: Awareness gaps, HIV, Noninfectious comorbidities, NICMs, Patient-reported outcomes, Survey data

Posted Date: January 15th, 2021

DOI: https://doi.org/10.21203/rs.3.rs-135380/v1

License: (c) (i) This work is licensed under a Creative Commons Attribution 4.0 International License. Read Full License 


\section{Abstract}

Background: This study was performed to investigate the gaps among patient-reported data, physicianreported data, and laboratory test data in human immunodeficiency virus (HIV)-infected patients and to detect potential risk factors for the awareness gap.

Methods: We included patients from 38 hospitals in China. Patient-reported data, physician-reported data, and laboratory test data on four typical noninfectious comorbidities (NICMs) (osteoporosis, kidney disease, neuropsychiatric comorbidities, and atherosclerotic cardiovascular disease (ASCVD)) were collected. We compared differences in and consistency of these types of data and investigated the associations between baseline characteristics and the awareness gap between patients and physicians.

Results: This study included 1390 patients. The difference between the diagnosis rate and laboratory test positivity rate for all NICMs was significant. Patient-reported data on the three NICMs other than kidney disease were significantly different from the diagnosis rates. The agreement between patients and physicians for all NICMs was poor. The region, infection stage, hospital level, sex, and age influenced the awareness gap between patients and physicians.

Conclusions: Our findings suggest that NICMs in HIV-infected patients in China are underestimated; gaps among patient awareness of disease exist, the clinical diagnosis rate, and the actual prevalence; patient characteristics influence the awareness gap between patients and physicians.

\section{Background}

The prevalence of human immunodeficiency virus/acquired immune deficiency syndrome (HIV/AIDS), which is a complex chronic disease, still imposes a considerable burden on the medical system, especially in developing countries ${ }^{1-3}$. The growing HIV-infected population is still a challenge for the Chinese medical system ${ }^{4}$. The incidence of HIV infection in China increased to 4.2 per 100000 individuals in 2017 from 0.23 per 100000 in $2004^{5}$. In addition, the HIV-infected population is also living longer, and the number of middle-aged and older people who are newly infected with HIV is rising rapidly 6,7 .

The introduction of combination antiretroviral therapy (ART) has contributed substantially to the reductions in morbidity and mortality associated with HIV infection ${ }^{8,9}$. Despite the increased life expectancy, mortality remains 3- to 15 -fold higher in HIV-infected people than in the general population ${ }^{9}$, 10. Together with the increased life expectancy of HIV-positive patients and the expansion of the elderly HIV-infected population, long-term treatment toxicity and old age-associated comorbid conditions have become more prevalent in these patients ${ }^{11-13}$. In addition, the progression of HIV infection may exacerbate or cause comorbidities. Although diseases caused by immunodeficiency contribute some to the excess mortality observed in the HIV-infected population, noninfectious comorbidities (NICMs) lead to more than half of the deaths among HIV-infected patients who undergo ART ${ }^{14,15}$. Renal failure, 
osteoporosis, neuropsychiatric comorbidities, and cardiovascular disease (CVD) are representative NICMS among HIV-infected patients, as these conditions have relatively high prevalences in HIV-infected patients ${ }^{8,15-17}$.

Research is needed to determine whether these comorbidities are underestimated among HIV populations. Insufficient awareness on the part of both doctors and patients may lead to the underestimation of NICMs and the absence of related diagnosis and treatment, which in turn may lead to increased mortality and decreased patient quality of life ${ }^{10,18}$. In addition, this kind of research is particularly lacking in the Asian HIV-infected population. Therefore, research focusing on the differences among the patient awareness, clinical diagnosis, and prevalence of NICMs based on national survey data of HIV-infected patients is needed to fill this gap. To better understand the NICM awareness gap and the associated risk factors, this cross-sectional study was conducted. This study explored the gap between patient-reported data and physician-reported data and the gap between the clinical diagnosis rate and laboratory test positivity rate.

\section{Methods}

\section{Study Design And Participants}

A national survey was conducted in 38 hospitals. In total, 139 physicians from the departments of infectious diseases in these hospitals were selected; then, ten patients seen by each physician were selected randomly. The inclusion criteria for the patients were as follows: (1) use of ART and (2) ability to understand and complete the questionnaire (including four self-assessment scales for osteoporosis, depression, anxiety, and sleep disorders). The questionnaire used in this survey included baseline information and information on comorbid conditions, and it was completed by both the physician and the patient. The questionnaire was paper-based, and the involved doctors interviewed their own patients and completed the questionnaire based on the interview. This study was evaluated and approved by the ethics committee of Shanghai Public Health Clinical Center. Written informed consent was obtained from all patients and doctors before the survey began.

\section{Noninfectious Comorbidities}

Four classes of common noninfectious comorbidities, namely, osteoporosis, kidney disease, neuropsychiatric comorbidities, and atherosclerotic cardiovascular disease (ASCVD), were investigated in this study. Patient-reported data, physician-reported data, and laboratory test data on the above four groups of diseases were collected via the questionnaire, and we further summarized them into 12 binary variables. Details are as follows:

\section{Osteoporosis}


The patient self-reported osteoporosis risk was measured by the IOF Osteoporosis Risk Check ${ }^{19,20}$. Physician-reported osteoporosis was measured by the diagnosis records regarding osteoporosis and related diseases. The laboratory indicator (gold standard) of osteoporosis was bone mineral density in the lumbar spine and hip (within the last 6 to 12 months).

\section{Kidney diseases}

Self-reported kidney disease was indicated by the patient-reported chronic kidney disease stage. The physician-reported metric was the diagnostic record for kidney diseases. The laboratory indicators of kidney disease were the blood urea nitrogen and creatinine levels. ${ }^{21-23}$

\section{Neuropsychiatric comorbidities}

The neuropsychiatric comorbidities measured in this survey were anxiety, depression, and sleep disorders 24,25 . Self-reported neuropsychiatric comorbidities were measured by the Chinese versions of three selfrated scales for anxiety ${ }^{26}$, depression ${ }^{27}$, and sleep disorders ${ }^{28}$. Physician-reported neuropsychiatric comorbidities were obtained from the diagnostic records of the above neuropsychiatric comorbidities.

\section{ASCVD}

The self-reported ASCVD risk was determined by the patient-reported ASCVD risk factors. Physicianreported ASCVD was obtained from the diagnostic records of cardiovascular diseases. The laboratory indicator for ASCVD was the 10-year risk of ASCVD, calculated based on blood pressure, diabetes history, total cholesterol (TC), low-density lipoprotein cholesterol (LDL-C), and high-density lipoprotein cholesterol (HDL-C) ${ }^{29,30}$.

\section{Statistical analysis}

$\mathrm{R}$ language (version 3.6.2) was used for all analyses. Proportions or mean values and standard deviations were used for the descriptive analysis of the baseline characteristics. Comparisons between two groups were made using chi-square tests (a 2-tailed probability value of 0.05 was considered significant). The consistency of the two measurements for the same disease was determined with Cohen's kappa coefficient ${ }^{31}$. An awareness gap was defined by an inconsistency in the information reported by physicians and patients regarding noninfectious comorbidities. Multiple logistic regression analysis was applied to detect potential associations between background characteristics and awareness gaps. The associations were considered statistically significant at the $P<0.05$ level.

\section{Results}

\section{Baseline characteristics}

There were 139 physicians and 1390 HIV-infected patients who eventually participated in the survey. As shown in Table 1, nearly half of the participants were from the eastern region of China (45.3\%), followed 
by the western region (38.8\%); the lowest proportion of participants was from the central region (15.8\%). A total of $95.0 \%$ of the participants were from tertiary hospitals. The average age of the patients was 48.8 (13.8) years old. Most patients were male (79.9\%). The average body mass index of patients was $22.2 \mathrm{~kg} / \mathrm{m}^{2}$ (3.6). More than half of the patients were in the clinical latency stage (59.2\%); $40.4 \%$ of patients were in the AIDS stage; and only $0.4 \%$ of patients were in the acute HIV infection stage. More than half of the patients were on urban employee basic medical insurance $(62.1 \%) ; 21.4 \%$ of patients were on the new rural cooperative medical scheme; $16.5 \%$ of patients were self-funded; and only $0.2 \%$ of patients were on commercial insurance. A total of $97.6 \%$ of the patients were included in the Integrated AIDS Information System. 
Table 1

Baseline characteristics of enrolled doctors and patients, china

\begin{tabular}{|c|c|}
\hline Characteristics & $\begin{array}{l}\% \text { or } \\
\text { Mean (Standard Deviation) }\end{array}$ \\
\hline \multicolumn{2}{|l|}{ Doctor $(n=139)$} \\
\hline \multicolumn{2}{|l|}{ Region } \\
\hline East & 45.3 \\
\hline Central & 15.8 \\
\hline West & 38.8 \\
\hline \multicolumn{2}{|l|}{ Hospital Level } \\
\hline Tertiary & 95.0 \\
\hline Others & 5.0 \\
\hline \multicolumn{2}{|l|}{ Patients $(n=1390)$} \\
\hline Age, years & $48.8(13.8)$ \\
\hline \multicolumn{2}{|l|}{ Sex } \\
\hline Male & 79.9 \\
\hline Female & 20.1 \\
\hline BMI & $22.2(3.6)$ \\
\hline \multicolumn{2}{|l|}{ Stages of HIV Infection } \\
\hline Acute HIV Infection & 0.4 \\
\hline Clinical Latency & 59.2 \\
\hline AIDS & 40.4 \\
\hline \multicolumn{2}{|l|}{ Medical Insurance } \\
\hline Urban Employee Basic Medical Insurance & 62.1 \\
\hline New Rural Cooperative Medical Scheme & 21.4 \\
\hline Self-Funded & 16.5 \\
\hline Commercial Insurance & 0.2 \\
\hline Included in "Integrated AIDS Information System" & 97.6 \\
\hline
\end{tabular}

\section{Comparison And Consistency In Different Nicms}


Figure 1 shows a comparison among patient-reported results, physician-reported results, and laboratory indicators. The proportion of patients with self-reported existing osteoporosis disease risks was $83.6 \%$, and the physician diagnosis rate was only $0.7 \%$. Only 37 patients underwent a bone density test, and the proportion of those patients who had osteoporosis was $16.2 \%$. There was a significant difference between patient-reported disease risk and the diagnosis rate $(P<0.001)$; the difference between the diagnosis rate and laboratory test results was also significant $(P<0.001)$. The proportion of patients who reported kidney diseases was $3.8 \%$; the physician-reported diagnosis rate was $5.4 \%$; and the rate of positivity on laboratory tests was $11.3 \%$. The difference between the patient-reported disease risk and the physician-reported diagnosis rate for kidney diseases was nonsignificant $(P=0.052)$, while the difference between the diagnosis rate and the laboratory test results was significant $(P<0.001)$. The proportion of patients who reported neuropsychiatric comorbidities was $66.0 \%$, and the diagnosis rate was $16.0 \%$. No related laboratory test indexes for neuropsychiatric comorbidities were investigated in this study. There was a significant difference between the patient-reported disease risk and physician-reported diagnosis rate $(P<0.001)$. The proportion of patients who reported ASCVD was $25.7 \%$; the diagnosis rate was $4.2 \%$; and the rate of positivity on laboratory tests was $42.3 \%$. The patient-reported disease risk for ASCVD was significantly different from the physician-reported diagnosis rate $(P<0.0001)$, and the difference between the diagnosis rate and the laboratory test results for ASCVD was also significant $(P<0.001)$. In addition, the differences among patient-reported results, physician-reported results, and laboratory indicators for osteoporosis, kidney diseases and ASCVD were all significant (all $P<0.001$ ).

To assess the interrater reliability of the patient-reported results and the physician-reported clinical diagnosis results for the same disease, we also calculated the Kappa statistic (as shown in Table 2). We found that the patient-reported results and physician-reported results were more similar for kidney disease than for the other three groups of diseases; while the percentage agreement was the same, the percentage agreement that would occur 'by chance' was significantly larger for kidney disease $(0.395$ compared to $0.001,0.170$, and 0.075 ). However, according to Landis and $\mathrm{Koch}^{31}$, these results showed that the agreement between patients and physicians with regard to all four diseases was poor. The agreement with regard to osteoporosis, neuropsychiatric comorbidities, and ASCVD was slight, and the agreement with regard to kidney diseases was fair.

Table 2

Cohen's kappa coefficient for comorbidities

\begin{tabular}{|lllllllllll|}
\hline & Comorbidity & \multicolumn{2}{l}{ Osteoporosis } & $\begin{array}{l}\text { Kidney } \\
\text { Disease }\end{array}$ & $\begin{array}{l}\text { Neuropsychiatric } \\
\text { Comorbidities }\end{array}$ & ASCVD \\
\hline & & Yes & No & Yes & No & Yes & No & Yes & No \\
\hline & 9 & 1 & 27 & 48 & 218 & 4 & 29 & 30 \\
\hline $\begin{array}{l}\text { Physician- } \\
\text { Reported } \\
\text { Diagnosis }\end{array}$ & Yes & No & 1153 & 227 & 26 & 1289 & 697 & 471 & 318 & 1003 \\
Cohen's Kappa & 0.001 & & 0.395 & 0.170 & & 0.075 \\
\hline
\end{tabular}




\section{Potential Risk Factors And Nicm Awareness Gaps}

Table 3 shows the results for the associations between potential risk factors and the awareness gap between patients and physicians for the four NICMs included in this study based on the results of multiple logistic regressions. Patients from the central and western regions were more likely to report results that were inconsistent with those reported by their physician than were patients from the eastern region with regard to osteoporosis (odds ratio $(\mathrm{OR})=1.96,95 \%$ confidence interval $(\mathrm{Cl}): 1.25,3.07$; OR $3.45,95 \% \mathrm{Cl}: 2.41,4.95$; respectively) and neuropsychiatric comorbidities ( $\mathrm{OR}=2.69,95 \% \mathrm{Cl}$ : 1.89, 3.81; $\mathrm{OR}=2.12,95 \% \mathrm{Cl}: 1.66,2.71$; respectively). Patients from tertiary hospitals were less likely to report information that was inconsistent with that reported by their physicians with regard to neuropsychiatric comorbidities ( $\mathrm{OR}=0.48,95 \% \mathrm{Cl}: 0.20,0.89)$. Old age (age $\geq 60)$ increased the risk of awareness gaps with regard to osteoporosis $(\mathrm{OR}=2.37,95 \% \mathrm{Cl}: 1.53,3.67)$, kidney disease $(\mathrm{OR}=3.71,95 \% \mathrm{Cl}: 2.32,5.93)$ and ASCVD (OR $=3.11,95 \% \mathrm{Cl}: 2.36,4.10)$. Male patients were less likely to report information that was inconsistent this that reported by their physicians with regard to kidney diseases $(\mathrm{OR}=0.48,95 \% \mathrm{Cl}: 0.29$, 0.79). Patients with acute HIV infections and those in the clinical latency stage were less likely to report results that were inconsistent with those of their physician compared with patients with AIDS with regard to kidney diseases $(\mathrm{OR}=0.04,95 \% \mathrm{Cl}: 0.01,0.16 ; \mathrm{OR}=0.04,95 \% \mathrm{Cl}: 0.01,0.18$; respectively $)$ and ASCVD $(\mathrm{OR}=0.34,95 \% \mathrm{Cl}: 0.14,0.86 ; \mathrm{OR}=0.34,95 \% \mathrm{Cl}: 0.13,0.86$; respectively). Additionally, inclusion in the "Integrated AIDS Information System" was a protective factor against an awareness gap with regard to ASCVD (OR $=0.43,95 \% \mathrm{Cl}: 0.22,0.86)$. 
Table 3

Multiple logistic regression analysis of risk factors for nicms awareness gaps between doctors and patients

\begin{tabular}{|c|c|c|c|c|c|c|c|c|}
\hline \multirow{2}{*}{$\begin{array}{l}\text { Characteristics } \\
(n=1390)\end{array}$} & \multicolumn{2}{|c|}{ Osteoporosis } & \multicolumn{2}{|c|}{ Kidney Disease } & \multicolumn{2}{|c|}{$\begin{array}{l}\text { Neuropsychiatric } \\
\text { Comorbidities }\end{array}$} & \multicolumn{2}{|l|}{ ASCVD } \\
\hline & OR & $95 \% \mathrm{Cl}$ & OR & $\begin{array}{l}95 \% \\
\mathrm{Cl}\end{array}$ & OR & $95 \% \mathrm{Cl}$ & OR & $\begin{array}{l}95 \% \\
\mathrm{Cl}\end{array}$ \\
\hline \multicolumn{9}{|l|}{$\begin{array}{l}\text { Region (East as } \\
\text { reference) }\end{array}$} \\
\hline Central & $1.96^{\star *}$ & $\begin{array}{l}1.25 \\
3.07\end{array}$ & 0.95 & $\begin{array}{l}0.43 \\
2.06\end{array}$ & $2.69^{\star \star \star}$ & $\begin{array}{l}1.89 \\
3.81\end{array}$ & 0.72 & $\begin{array}{l}0.48, \\
1.07\end{array}$ \\
\hline West & $3.45^{\star \star \star}$ & $\begin{array}{l}2.41 \\
4.95\end{array}$ & 1.47 & $\begin{array}{l}0.89 \\
2.43\end{array}$ & $2.12^{\star \star \star}$ & $\begin{array}{l}1.66 \\
2.71\end{array}$ & 0.81 & $\begin{array}{l}0.61 \\
1.07\end{array}$ \\
\hline Tertiary Hospital & 0.49 & $\begin{array}{l}0.20 \\
1.23\end{array}$ & 3.50 & $\begin{array}{l}0.87 \\
14.01\end{array}$ & $0.48^{* *}$ & $\begin{array}{l}0.20 \\
0.89\end{array}$ & 1.33 & $\begin{array}{l}0.70 \\
2.51\end{array}$ \\
\hline $\begin{array}{l}\text { Old Age (Age } \geq \\
60 \text { years) }\end{array}$ & $2.37^{\star \star \star}$ & $\begin{array}{l}1.53 \\
3.67\end{array}$ & $3.71^{\star \star \star}$ & $\begin{array}{l}2.32 \\
5.93\end{array}$ & 0.97 & $\begin{array}{l}1.53 \\
1.27\end{array}$ & $3.11^{\star \star \star}$ & $\begin{array}{l}2.36, \\
4.10\end{array}$ \\
\hline Male & 1.00 & $\begin{array}{l}0.68 \\
1.47\end{array}$ & $0.48^{\star \star}$ & $\begin{array}{l}0.29 \\
0.79\end{array}$ & 0.78 & $\begin{array}{l}0.68 \\
1.03\end{array}$ & 1.27 & $\begin{array}{l}0.92, \\
1.76\end{array}$ \\
\hline $\begin{array}{l}\text { Overweight (BMI } \geq \\
24 \mathrm{~kg} / \mathrm{m}^{2} \text { ) }\end{array}$ & 1.30 & $\begin{array}{l}0.92 \\
1.82\end{array}$ & 1.50 & $\begin{array}{l}0.90 \\
2.51\end{array}$ & 0.96 & $\begin{array}{l}0.92 \\
1.24\end{array}$ & 1.28 & $\begin{array}{l}0.97 \\
1.70\end{array}$ \\
\hline \multicolumn{9}{|l|}{$\begin{array}{l}\text { Stages of HIV } \\
\text { Infection (AIDS as } \\
\text { reference) }\end{array}$} \\
\hline Acute HIV Infection & 2.54 & $0.83,7.83$ & $0.04^{\star \star \star}$ & $\begin{array}{l}0.01 \\
0.16\end{array}$ & 1.26 & $\begin{array}{l}0.83 \\
3.08\end{array}$ & $0.34^{*}$ & $\begin{array}{l}0.14, \\
0.86\end{array}$ \\
\hline Clinical Latency & 2.67 & $\begin{array}{l}0.86 \\
8.27\end{array}$ & $0.04^{\star \star \star}$ & $\begin{array}{l}0.01 \\
0.18\end{array}$ & 1.35 & $\begin{array}{l}0.86 \\
3.32\end{array}$ & $0.34^{*}$ & $\begin{array}{l}0.13 \\
0.86\end{array}$ \\
\hline $\begin{array}{l}\text { With Any Medical } \\
\text { Insurance }\end{array}$ & 1.20 & $\begin{array}{l}0.84 \\
1.71\end{array}$ & 0.84 & $\begin{array}{l}0.45 \\
1.57\end{array}$ & 0.86 & $\begin{array}{l}0.84 \\
1.16\end{array}$ & 1.17 & $\begin{array}{l}0.83 \\
1.66\end{array}$ \\
\hline $\begin{array}{l}\text { Included in } \\
\text { "Integrated AIDS } \\
\text { Information System" }\end{array}$ & 1.63 & $\begin{array}{l}0.75 \\
3.55\end{array}$ & 0.42 & $\begin{array}{l}0.14 \\
1.24\end{array}$ & 1.86 & $\begin{array}{l}0.75 \\
3.65\end{array}$ & $0.43^{*}$ & $\begin{array}{l}0.22, \\
0.86\end{array}$ \\
\hline \multicolumn{9}{|c|}{${ }^{\star} P<0.05 ;{ }^{* \star} P<0.01 ; ;{ }^{* \star} P<0.001}$. \\
\hline$\star \star \star: P<0.001$ & & & & & & & & \\
\hline
\end{tabular}

\section{Discussion}

The prevalence of HIV/AIDS still imposes a substantial burden on the Chinese medical system because it is a complex chronic disease, and the HIV-infected population is still increasing. With aging and the 
increasing life expectancy of the HIV-positive population, as well as the rapidly increasing number of newly infected middle-aged and older adults, the toxicity associated with long-term treatment and old age-associated comorbidities are becoming more prevalent in patients. There have been few relevant studies on the awareness of NICMs and the gaps among the self-awareness of diseases, the diagnosis rate, and the prevalence according to laboratory indicators in HIV-infected patients, especially in the Chinese population. Therefore, to provide evidence to support clinical work, we conducted this study. In this study, patient-reported data, physician-reported data (the diagnosis of related diseases), and laboratory test results were compared. We found that the patient-reported risks of osteoporosis, neuropsychiatric comorbidities, and ASCVD were significantly different from the diagnosis rates; the difference between the patient-reported disease risk and diagnosis rate for kidney disease was nonsignificant. The differences between the diagnosis rate and rate of positivity on laboratory tests for all four groups of diseases were statistically significant. The differences among the above three disease assessment methods for all four groups of diseases were also statistically significant. In addition, we also assessed the interrater reliability of the patient-reported results and the physician-reported clinical diagnoses for the same diseases. We found that the agreement between patients and physicians with regard to all of the above four diseases was poor (kappa $<0.400)$. The agreement with regard to osteoporosis, neuropsychiatric comorbidities, and ASCVD was slight, and the agreement with regard to kidney diseases was fair.

To further analyze the findings we obtained and place them in context, we reviewed the prevalence of NICMs in the HIV-infected population in China and provided further interpretation. A limited number of studies have focused on osteoporosis in HIV-infected Chinese patients. Hsieh et al. ${ }^{32}$ conducted a multicenter study with $263 \mathrm{HIV}$-infected patients in China, and they found that $10.7 \%$ of patients reported a fracture history (though the majority of the cases were linked to trauma). Guo et al. ${ }^{33}$ observed lower bone mineral density in HIV-infected patients than in noninfected controls. In this study, we found a high self-reported risk (83.6\%) of osteoporosis in our sample, and the rate of a positive identification of osteoporosis in the patients who underwent a bone density test was $16.2 \%$. However, the diagnosis rate was only $0.7 \%$. This result may be due to the physicians' lack of awareness of this group of diseases, which stems from the fact that osteoporosis is usually not significant or fatal when it occurs. This may also explain the extremely low proportion of patients in our sample who underwent bone density tests, which in turn further reduced the rate of diagnosis of osteoporosis.

The incidence of chronic kidney disease is high in Chinese HIV-infected patients. A cross-sectional study with HIV-infected ART-naïve patients reported a prevalence of $16.1 \%^{34}$, and another cross-sectional study with 322 Chinese patients reported a prevalence of $16.8 \%{ }^{35}$. In our sample, the prevalence of kidney diseases was lower than but relatively close to the prevalence in the general Chinese population (10.8\% $(10.2-11.3))^{21}$. This difference may be related to the fact that compared with this study, the studies mentioned above were based on relatively small samples from large tertiary hospitals. As a result, the patients in their sample may have had more severe disease and have undergone ART for a longer time than a nationally representative sample. We also noticed that the self-report rate and diagnostic rate for 
kidney disease were relatively low, which may indicate that kidney disease is underdiagnosed in HIVinfected patients.

We used depression, sleep disorders, and anxiety to assess neuropsychiatric comorbidities in our patients. In previous studies, the prevalence of neuropsychiatric comorbidities has usually been assessed by patient self-reported scales, and the results of those studies were consistent with those of this study $(66.0 \%)^{36-38}$. However, in clinical practice, the rate of diagnosis of neuropsychiatric comorbidities is much lower. In this study, we found that only $16.0 \%$ of patients were diagnosed with neuropsychiatric comorbidities. This may be attributed to physicians' insufficient recognition of disease symptoms and the shortage of psychiatrists.

The prevalence of ASCVD in HIV-infected patients was $20.41 \%$ according to a meta-analysis of real-life data ${ }^{39}$, which is close to the patient-reported prevalence in this study (25.7\%); however, we did not find much information on the prevalence of ASCVD specifically in Chinese HIV-positive patients. As the physician-reported ASCVD metric reflected diagnosed cardiovascular disease, the proportion was lower than the patient-reported result ( $4.2 \%$ vs. $25.7 \%$ ). Moreover, the low diagnosis rate of ASCVD and the rate of positive laboratory test results of nearly $50 \%$ can be attributed to misclassification. More details are described in the limitations section.

We also identified several potential risk factors for NICM awareness gaps. We found that receiving treatment in hospitals in the eastern region was a protective factor against awareness gaps between patients and physicians with regard to osteoporosis and neuropsychiatric comorbidities, while receiving treatment in tertiary hospitals was a protective factor against awareness gaps with regard to neuropsychiatric comorbidities. This may be due to the greater amount of medical resources in the eastern region and tertiary hospitals (including but not limited to the number of specialists and testing equipment). Old age (age $\geq 60$ ) was a risk factor for awareness gaps with regard to osteoporosis, kidney disease and ASCVD. This may be related to the increased prevalence of these diseases with increasing age. Patients with acute HIV infections and in the clinical latency stage were less likely to report results that were inconsistent with those reported by their physician than patients with AIDS with regard to kidney diseases and ASCVD. This may be related to the side effects of long-term ART in AIDS patients.

There are several limitations of our study. First, the hospitals included in this study were selected and not randomly sampled (though the patients were selected randomly). In our sample, the proportion of patients from the central region was slightly lower than the proportions of patients from the other regions, and this sample was not nationally represented. However, compared with current published studies on NICMs in Chinese HIV-infected patients (for example, ${ }^{38,40,41}$ ), the sample in our study was larger and was drawn from all regions of the country, suggesting that our sample is relatively more representative of HIV-infected patients in China. Second, there was a significant difference in the measurement standards for ASCVD between physician-reported data and the laboratory test results. The physician-reported data on ASCVD were the records of the diagnosis of cardiovascular diseases. In contrast, a positive laboratory test result indicated a moderate or greater 10-year ASCVD risk (which included existing cardiovascular 
diseases and additional ASCVD risk). Despite these limitations, this study has some implications for clinical work.

\section{Conclusion}

Generally, NICMs in Chinese HIV-infected patients were underestimated. HIV-infected patients are usually treated in the department of infectious diseases, and infectious disease specialists may not identify these common noninfectious comorbidities in a timely manner or provide the appropriate treatment to the patients. Therefore, it is important for infectious disease specialists to pay more attention to possible noninfectious comorbid conditions. In addition, patient self-reported scales can be introduced for the primary screening of osteoporosis and neuropsychiatric comorbidities. In addition, using a multidisciplinary team to treat HIV patients could be a reasonable solution to this issue. In this way, doctors would be likely to identify comorbidities earlier and adjust the treatment plan of patients as soon as possible.

Our findings suggest that NICMs in HIV-infected patients in China are underestimated, and there are gaps among patient awareness of disease, the clinical diagnosis rate, and the actual prevalence. The awareness gap between patients and physicians is influenced by patient characteristics.

\section{Abbreviations}

AIDS: Acquired immune deficiency syndrome; ART: Antiretroviral therapy; ASCVD: Atherosclerotic cardiovascular disease; Cl: Confidence interval; CVD: Cardiovascular disease; HDL-C: High-density lipoprotein cholesterol; HIV: Human immunodeficiency virus; LDL-C: Low-density lipoprotein cholesterol; NICMs: Noninfectious comorbidities; OR: Odds ratio; TC: Total cholesterol

\section{Declarations}

\section{Ethics approval and consent to participate}

The study was conducted in accordance with Declaration of Helsinki and ethically approved by the Ethics Committee of Shanghai Public Health Clinical Center. Written informed consent was obtained from all patients and doctors before the survey began.

\section{Consent for publication}

Not applicable.

\section{Availability of data and materials}

All the data supporting our findings are contained within this article.

\section{Competing interests}


The authors declare that they have no competing interests.

\section{Funding}

This work was supported by the Ministry of Science and Technology, the People's Republic of China (NO: 2017ZX09304027).

\section{Authors' Contributions}

The project was completed by the HIV Comorbidity Management Working Group. All authors contributed substantively to this study and article. Hongzhou Lu was the leader of the project and was responsible for project development, and Jun Chen contributed to the data analysis and manuscript writing.

\section{Acknowledgements}

We would like to thank all members who offered assistance during the study.

HIV Comorbidity Management Working Group [HCMWG] ${ }^{\#}$

Jun Chen ${ }^{1}$, Shuiqing Liu ${ }^{2}$, Jun Liu ${ }^{3}$, Haibo Ding ${ }^{4}$, Rugang Wang ${ }^{5}$, Zhihao Meng ${ }^{6}$, Peng Zhang ${ }^{7}$, Yinghua Wei $^{8}$, Lijing Wang ${ }^{9}$, Jie Yang ${ }^{10}$, Jian Zhang ${ }^{11}$, Qingxia Zhao ${ }^{12}$, Xiaohong Chen ${ }^{13}$, Ruzhi Ou ${ }^{14}$, Yuanhong $\mathrm{He}^{15}$, Yahong Chen ${ }^{16}$, Juan Chen ${ }^{17}$, Min Wang ${ }^{18}$, Biao Zhu ${ }^{19}$, Yaokai Chen ${ }^{20}$, Juan $\mathrm{Jin}^{21}$, An Liu ${ }^{22}$, Yang Zhou $^{23}$, Guijv Gao ${ }^{24}$, Ping Ma ${ }^{25}$, Hongxia Wei ${ }^{26}$, Feifei $\mathrm{Su}^{27}$, Lianguo Ruan ${ }^{28}$, Tianjun Jiang ${ }^{29}$, Nanhong Zheng ${ }^{30}$, Jianhua $\mathrm{Yu}^{31}$, Guangyong $\mathrm{Xu}^{32}$, Feng Qian ${ }^{33}$, Cenmian Huang ${ }^{34}$, Lingyao $\mathrm{Du}^{35}$, Na Wang ${ }^{36}$, Huiling Zhou ${ }^{37}$, Jie Peng ${ }^{38}$, Hongzhou Lu ${ }^{1}$

${ }^{1}$ Department of Infectious Diseases, Shanghai Public Health Clinical Center, Fudan University, Shanghai, 201508, People's Republic of China

${ }^{2}$ Department of Infectious Diseases, the Fifth People's Hospital of Guiyang, Guiyang, Guizhou, 550004, China

${ }^{3}$ Department of HIV/AIDS, The Third People's Hospital of Kunming, Kunming, 650041, China

${ }^{4}$ Key Laboratory of AIDS Immunology of National Health and Family Planning Commission, Department of Laboratory Medicine, The First Affiliated Hospital, China Medical University, Shenyang, China

${ }^{5}$ Affiliated Dalian Sixth People's Hospital, Dalian Medical University, Dalian 116031, China

${ }^{6}$ Infectious Diseases Department, Longtan Hospital of Guangxi, Liuzhou, Guangxi 545005 China

${ }^{7}$ The Infectious Diseases Department, the Fifth Affiliated Hospital of Guangxi Medical University (the People's Hospital of Liuzhou City), Liuzhou 545006, Guangxi, China 
${ }^{8}$ Department of Infectious Disease and Department of Pediatrics, the First Affiliated Hospital of Guangxi Medical University, Nanning 530021, China

${ }^{9}$ The Fifth Hospital of Shijiazhuang, Shijiazhuang, Hebei, China

${ }^{10}$ Yunnan AIDS Care Center (YNACC), Yunnan Provincial Infectious Disease Hospital, Kunming 650500, China

${ }^{11}$ Department of Infectious Diseases, Changchun Infectious Disease Hospital, Changchun, China

${ }^{12}$ Department of Infection, Zhengzhou Sixth People's Hospital, Zhengzhou, China

${ }^{13}$ Department of Infectious Diseases, The Fourth Affiliated Hospital of Harbin Medical University, Harbin, China

${ }^{14}$ Department of Infection, the Fourth People's Hospital of Nanning, Nanning 530023, China

${ }^{15}$ Department of Infection, Public Health Clinical Center of Chengdu, Sichuan Province, China

${ }^{16}$ Department of Infectious Diseases, Mengchao Hepatobiliary Hospital, Fujian Medical University, Fuzhou, Fujian, China; Fujian HIV/AIDS Diagnosis and Treatment Center, Fuzhou, Fujian, China

${ }^{17}$ Department of Infectious Disease, The Second People's Hospital of Yancheng City, Yancheng, China ${ }^{18}$ The First Hospital of Changsha, Changsha, China

${ }^{19}$ Department of Infectious Diseases, State Key Laboratory for the Diagnosis and Treatment of Infectious Diseases, Collaborative Innovation Center for the Diagnosis and Treatment of Infectious Diseases, the First Affiliated Hospital, College of Medicine, Zhejiang University, Hangzhou, China

${ }^{20}$ Department of Infectious Diseases, Chongqing Public Health Medical Center, Chongqing, China

${ }^{21}$ The eighth hospital of Xi'an, Xi'an, China

${ }^{22}$ Beijing Youan Hospital, Capital Medical University

${ }^{23}$ HKU-AIDS Institute Shenzhen Research Laboratory, Shenzhen Key Laboratory of Infection and Immunity, the Key Laboratory for Diagnosis and Treatment of Emerging Infectious Diseases in Guangdong, National Clinical Research center for Infectious Diseases, The Third People's Hospital of Shenzhen, Shenzhen, China

${ }^{24}$ Beijing Ditan Hospital, Capital Medical University

${ }^{25}$ Tianjin Second People's Hospital 
${ }^{26}$ Department of Infectious Disease, the Second Hospital of Nanjing, Nanjing University of Chinese Medicine, 1-1 Zhongfu Road, Nanjing, 210003, China

${ }^{27}$ Department of Infectious Diseases, Wenzhou Central Hospital, Zhejiang, China

${ }^{28}$ Department of Infectious Diseases, Wuhan Medical Treatment Center, Wuhan, Hubei, 430023, China

${ }^{29}$ The Fifth Medical Center of PLAGH

${ }^{30}$ Ningbo No 2 Hospital, Ningbo, Zhejiang, China

${ }^{31}$ Department of Infectious Diseases, XIXI Hospital of Hangzhou, Hangzhou, China

32Dermatological department, Qingdao Infectious Diseases Hospital, Qingdao, China

${ }^{33}$ Division of HIV-1/AIDS, The Fifth People's Hospital of Suzhou, Suzhou, 215000, China

${ }^{34}$ The Infectious Diseases Hospital of Gejiu city Yunnan Province, Gejiu, China

${ }^{35}$ Division of Infectious Diseases, State Key Laboratory of Biotherapy and Center of Infectious Diseases, West China Hospital, Sichuan University, Chengdu, China

${ }^{36}$ Key Laboratory of Molecular Biology for Infectious Diseases (Ministry of Education), Institute for Viral Hepatitis, Department of Infectious Diseases, The Second Affiliated Hospital, Chongqing Medical University

${ }^{37}$ Department of Infectious Disease, The First Peoples' Hospital of Foshan, Foshan City, Guangdong Province 528000, People's Republic of China

${ }^{38}$ Department of Infectious Diseases, Nanfang Hospital, Southern Medical University, Guangzhou, China

\section{References}

1. Wu Z, Wang Y, Detels R, et al. HIV/AIDS in China: Epidemiology, Prevention and Treatment. Springer; 2019.

2. Campbell EM, Jia H, Shankar A, et al. Detailed transmission network analysis of a large opiate-driven outbreak of HIV infection in the United States. The Journal of infectious diseases 2017;216(9):105362.

3. Narayan KV, Miotti PG, Anand NP, et al. HIV and noncommunicable disease comorbidities in the era of antiretroviral therapy: a vital agenda for research in low-and middle-income country settings. LWW, 2014.

4. Zheng S. The growing threat of China's HIV epidemic. The Lancet Public Health 2018;3(7):e311. 
5. Qiao Y-c, Xu Y, Jiang D-x, et al. Epidemiological analyses of regional and age differences of HIV/AIDS prevalence in China, 2004-2016. International Journal of Infectious Diseases 2019;81:215-20.

6. Zhang Y, Fuller-Thomson E, Mitchell CA, et al. Older adults with HIV/AIDS in rural China. The open AIDS journal 2013;7:51.

7. Xie T, Wu N. Epidemiological and mortality analysis of older adults with HIV in eastern China. Clinical interventions in aging 2013;8:1519.

8. Roncero C, Barral C, Rodríguez-Cintas L, et al. Psychiatric comorbidities in opioid-dependent patients undergoing a replacement therapy programme in Spain: The PROTEUS study. Psychiatry Research 2016;243:174-81.

9. Goulet JL, Fultz SL, Rimland D, et al. Do patterns of comorbidity vary by HIV status, age, and HIV severity? Clinical infectious diseases 2007;45(12):1593-601.

10. Guaraldi G, Palella Jr FJ. Clinical implications of aging with HIV infection: perspectives and the future medical care agenda. Aids 2017;31:S129-S35.

11. Xiao J, Du S, Tian Y, et al. Causes of death among patients infected with HIV at a tertiary care hospital in China: An observational cohort study. AIDS research and human retroviruses 2016;32(8):782-90.

12. Guaraldi G, Zona S, Brothers TD, et al. Aging with HIV vs. HIV seroconversion at older age: a diverse population with distinct comorbidity profiles. PloS one 2015;10(4).

13. Stoff DM, Khalsa JH, Monjan A, et al. Introduction: HIV/AIDS and aging. LWW, 2004.

14. 14.

15. Guaraldi G, Orlando G, Zona S, et al. Premature age-related comorbidities among HIV-infected persons compared with the general population. Clinical infectious diseases 2011;53(11):1120-6.

16. Gunter J, Callens S, De Wit S, et al. Prevalence of non-infectious comorbidities in the HIV-positive population in Belgium: a multicenter, retrospective study. Acta Clinica Belgica 2018;73(1):50-3.

17. Moran CA, Weitzmann MN, Ofotokun I. Bone loss in HIV infection. Current treatment options in infectious diseases 2017;9(1):52-67.

18. Maciel RA, Klück HM, Durand $M$, et al. Comorbidity is more common and occurs earlier in persons living with HIV than in HIV-uninfected matched controls, aged 50 years and older: a cross-sectional study. International Journal of Infectious Diseases 2018;70:30-5.

19. Francis K, Matthews BL, Van Mechelen W, et al. Effectiveness of a community-based osteoporosis education and self-management course: a wait list controlled trial. Osteoporosis international 2009;20(9):1563-70.

20. Holzemer WL, Corless IB, Nokes KM, et al. Predictors of self-reported adherence in persons living with HIV disease. AIDS patient care and STDs 1999;13(3):185-97.

21. Zhang L, Wang F, Wang L, et al. Prevalence of chronic kidney disease in China: a cross-sectional survey. The Lancet 2012;379(9818):815-22. 
22. Calza L, Vanino E, Magistrelli E, et al. Prevalence of renal disease within an urban HIV-infected cohort in northern Italy. Clinical and experimental nephrology 2014;18(1):104-12.

23. Kong X, Ma Y, Chen J, et al. Evaluation of the Chronic Kidney Disease Epidemiology Collaboration equation for estimating glomerular filtration rate in the Chinese population. Nephrology Dialysis Transplantation 2013;28(3):641-51.

24. Aouizerat BE, Miaskowski CA, Gay C, et al. Risk factors and symptoms associated with pain in HIVinfected adults. Journal of the Association of Nurses in AIDS Care 2010;21(2):125-33.

25. Robbins JL, Phillips KD, Dudgeon WD, et al. Physiological and psychological correlates of sleep in HIV infection. Clinical Nursing Research 2004;13(1):33-52.

26. Lin MC, Endler NS, Kocovski NL. State and trait anxiety: A cross-cultural comparison of Chinese and Caucasian students in Canada. Current Psychology 2001;20(1):95-111.

27. Chin WY, Choi EP, Chan KT, et al. The psychometric properties of the Center for Epidemiologic Studies Depression Scale in Chinese primary care patients: factor structure, construct validity, reliability, sensitivity and responsiveness. PloS one 2015;10(8).

28. Tsai P-S, Wang S-Y, Wang M-Y, et al. Psychometric evaluation of the Chinese version of the Pittsburgh Sleep Quality Index (CPSQI) in primary insomnia and control subjects. Quality of Life Research 2005;14(8):1943-52.

29. Association CSoCoCM, Cardiology EBoCJo. Chinese guidelines for prevention of cardiovascular diseases. Zhonghua Xin Xue Guan Bing Za Zhi 2011;39(1):3.

30. Feinstein MJ, Hsue PY, Benjamin LA, et al. Characteristics, prevention, and management of cardiovascular disease in people living with HIV: a scientific statement from the American Heart Association. Circulation 2019;140(2):e98-e124.

31. Landis JR, Koch GG. An application of hierarchical kappa-type statistics in the assessment of majority agreement among multiple observers. Biometrics 1977:363-74.

32. Hsieh E, Fraenkel L, Bradley EH, et al. Osteoporosis knowledge, self-efficacy, and health beliefs among Chinese individuals with HIV. Archives of osteoporosis 2014;9(1):201.

33. Guo F, Yu X, Luo L, et al. The impact of highly active antiretroviral therapy on bone mineral density in human immunodeficiency virus infected patients. Zhonghua nei ke za zhi 2010;49(8):649-52.

34. Cao Y, Gong M, Han Y, et al. Prevalence and risk factors for chronic kidney disease among HIVinfected antiretroviral therapy-naïve patients in $M$ ainland $C$ hina: A multicenter cross-sectional study. Nephrology 2013;18(4):307-12.

35. Cheung CY, Wong KM, Lee MP, et al. Prevalence of chronic kidney disease in Chinese HIV-infected patients. Nephrology Dialysis Transplantation 2007;22(11):3186-90.

36. Wang Y-Y, Zhao J, Zhang Q, et al. Prevalence of depressive syndrome and their association with demographic and clinical characteristics in Chinese HIV patients. AIDS care 2018;30(11):1388-92.

37. Huang X, Li H, Meyers K, et al. Burden of sleep disturbances and associated risk factors: A crosssectional survey among HIV-infected persons on antiretroviral therapy across China. Scientific 
reports 2017;7(1):1-8.

38. Niu L, Luo D, Liu Y, et al. The mental health of people living with HIV in China, 1998-2014: a systematic review. PLoS One 2016;11(4).

39. Grand M, Bia D, Diaz A. Cardiovascular Risk Assessment in People Living With HIV: A Systematic Review and Meta-Analysis of Real-Life Data. Current HIV Research 2020;18(1):5-18.

40. Jiang H, Xie N, Cao B, et al. Determinants of progression to AIDS and death following HIV diagnosis: a retrospective cohort study in Wuhan, China. PloS one 2013;8(12).

41. Liu H, He X, Levy JA, et al. Psychological impacts among older and younger people living with HIV/AIDS in Nanning, China. Journal of aging research 2014;2014.

\section{Figures}

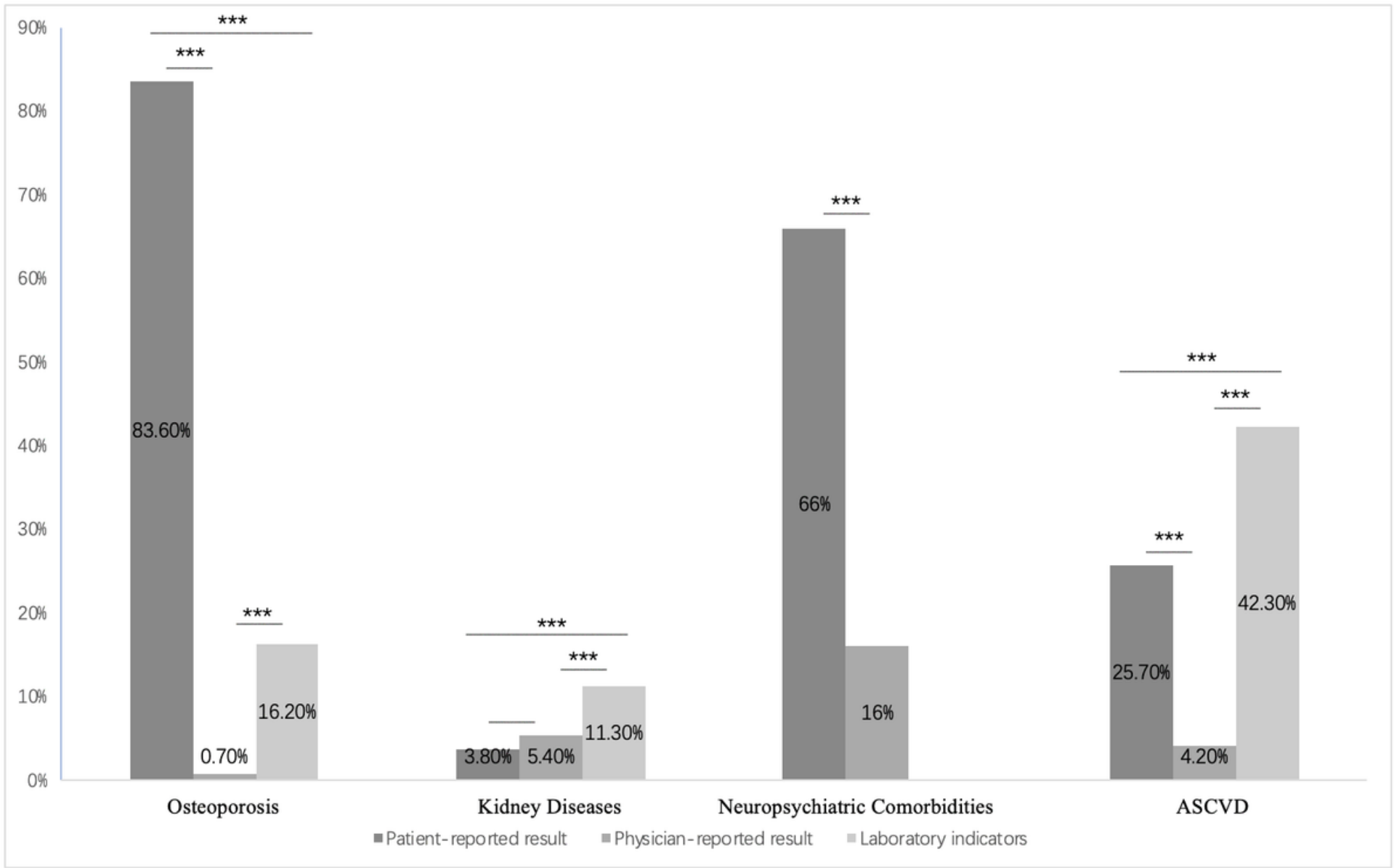

\section{Figure 1}

The comparison among patient-reported results, physician-reported results, and laboratory indicators. *ᄎ*: $\mathrm{P}<0.001$ 